Kevin Rostasy, MD

Barbara Bajer-Kornek, MD

Sunita Venkateswaran, MD

Cheryl Hemingway, MD

Marc Tardieu, MD, PhD

Correspondence to

Dr. Rostasy:

k.rostasy@kinderklinik-datteln.de

\title{
Differential diagnosis and evaluation in pediatric inflammatory demyelinating disorders
}

\section{ABSTRACT}

Major advances have been made in the clinical and radiologic characterization of children presenting with the different forms of an acquired inflammatory demyelinating syndrome (ADS) such as acute disseminating encephalomyelitis, neuromyelitis optica spectrum disorders, and clinically isolated syndromes. Nevertheless, a proportion of cases that present with similar symptoms are due to a broad spectrum of other inflammatory disorders affecting the white matter, primary CNS tumors, or neurometabolic diseases. The clinician therefore has to be aware of the different forms of ADS, the risk factors for a chronic-relapsing course, and features that indicate an alternative diagnosis. The goal of this article is therefore to provide an outline of a pathway for evaluating pediatric patients with a presumed inflammatory demyelinating disorder and discussing the spectrum of the more common differential diagnoses. Neurology ${ }^{\circledR}$ 2016;87 (Suppl 2):S28-S37

\section{GLOSSARY}

ADEM = acute disseminating encephalomyelitis; $\mathbf{A D S}$ = acquired demyelinating syndromes; $\mathbf{A N E}=$ acute necrotizing encephalopathy; AQP4 = aquaporin-4; BBE = Bickerstaff brainstem encephalitis; BBGD = biotin-responsive basal ganglia disease; $\mathbf{C I S}=$ clinically isolated syndromes; $\mathbf{D I S}=$ dissemination in space; DIT = dissemination in time; GBS = GuillainBarré syndrome; $\mathbf{H L H}=$ hemophagocytic lymphohistiocytosis; $\mathbf{I g G}=$ immunoglobulin G; LCH = Langerhans cell histiocytosis; LETM = longitudinally extensive transverse myelitis; MOG = myelin oligodendrocyte glycoprotein; MS = multiple sclerosis; NMOSD = neuromyelitis optica spectrum disorders; ON = optic neuritis; PACNS = primary angiitis of the CNS; PCNSL = primary CNS Iymphoma; PML = progressive multifocal leukoencephalopathy; $\mathbf{S L E}=$ systemic lupus erythematosus; $\mathbf{T M}=$ transverse myelitis.

Acquired demyelinating syndromes (ADS) represent a group of disorders such as acute disseminating encephalomyelitis (ADEM), neuromyelitis optica spectrum disorders (NMOSD), and clinically isolated syndromes (CIS). A timely and precise diagnosis is mandatory in order to ensure adequate patient management, but due to the extensive differential diagnosis, it is not uncommon that children are assigned an incorrect diagnosis.

The focus of this review is to describe and delineate the different subtypes of ADS from the broad spectrum of other inflammatory disorders of the white matter, genetic disorders affecting the innate and adaptive immune system, primary CNS tumors, and neurometabolic diseases.

ACQUIRED DEMYELINATING SYNDROMES The clinical picture of ADS comprises ADEM, characterized by polyfocal deficits and encephalopathy; CIS, characterized by monofocal or polyfocal deficits without encephalopathy; pediatric multiple sclerosis (MS); and NMOSD (table 1). ADS occur as a monophasic illness, but may also represent the onset of a chronic relapsing disorder.

Among the various forms of ADS, ADEM offers the widest spectrum of differential diagnosis. The clinical picture of ADEM is characterized by a polyfocal onset, associated with encephalopathy. ${ }^{1}$ Encephalopathy in $\mathrm{ADEM}$ is defined as a qualitative or quantitative disturbance of consciousness, such as irritability, somnolence, or coma, and must occur in the absence of fever. MRI is an important tool in distinguishing ADEM from other diseases and in particular from the first attack of MS. ${ }^{2}$ Lesions found on MRI are typically diffuse, ill-defined, $>1-2 \mathrm{~cm}$ in size, and in the cerebral white matter, but may also affect the thalamus, basal ganglia, and spinal cord. T1-hypointense lesions are rare. ${ }^{1}$ Among the patients presenting with ADS, ADEM is found more

From the Department of Pediatric Neurology (K.R.), Children's Hospital Datteln, University Witten/Herdecke, Germany; Department of Neurology (B.B.-K.), Medical University of Vienna, Austria; Division of Neurology (S.V.), Children's Hospital of Eastern Ontario, Ottawa, Canada; Pediatric Neurology (C.H.), Great Ormond Street Hospital for Children NHS Foundation Trust, London, UK; and National Reference Center for Inflammatory Diseases of the Brain (M.T.), Hôpitaux Universitaires Paris-Sud, University Paris-Sud, France.

Go to Neurology.org for full disclosures. Funding information and disclosures deemed relevant by the authors, if any, are provided at the end of the article. 
Conditions Consider following investigations (in addition to MRI brain/spine)

ADEM

CBC, liver enzymes, renal function, ESR, complement $(C 3, C 4)$, ANA, dsDNA, 25(OH) vitamin D, ferritin, triglycerides, ACE, AQP4, MOG antibodies; bacterial and viral studies as indicated (blood, throat swab, stool)

NMOSD

CSF studies with cell count, protein, glucose, oligoclonal bands, cytology, MRZ reaction; bacterial and viral studies as indicated

CIS

Evoked potentials (visual, somatosensory, auditory), ophthalmology with OCT, neuropsychological testing, urology, rheumatology

Abbreviations: $\mathrm{ACE}=$ angiotensin-converting enzyme; $\mathrm{ADEM}=$ acute disseminating encephalomyelitis; $\mathrm{ANA}=$ antinuclear antibodies; AQP4 = aquaporin-4; CBC = complete blood count; CIS = clinically isolated syndromes; dsDNA = double-stranded DNA; ESR = erythrocyte sedimentation rate; MRZ = measles, rubella, varicella zoster virus; OCT = optical coherence tomography; MOG = myelin oligodendrocyte glycoprotein; NMOSD = neuromyelitis optica spectrum disorders.

frequently in younger children. ${ }^{3}$ Typically ADEM is a monophasic disorder, but may also represent the first attack of pediatric MS. ${ }^{4}$

CIS are characterized by monofocal or polyfocal events without encephalopathy, unless explained by fever. ${ }^{1}$ The most common clinical presentations of CIS in children are optic neuritis $(\mathrm{ON})$, transverse myelitis (TM), or brainstem/cerebellar syndromes or syndromes attributed to the cerebral hemispheres. ${ }^{3,5}$ Unilateral $\mathrm{ON}$ is defined by acute/subacute and painful visual loss, impairment of visual acuity and color perception, MRI evidence of optic nerve swelling, or abnormal signal or enhancement. ${ }^{3}$ Bilateral $\mathrm{ON}$ is more common in ADEM or NMOSD. TM is characterized by acute/subacute onset of bilateral symmetric or asymmetric sensory or motor deficits, sometimes associated with sphincter dysfunction, with a defined sensory level, and MRI evidence of spinal cord swelling or abnormal signal intensity or enhancement. ${ }^{3}$ If brain MRI is normal at the time of a CIS, the risk of MS is low. ${ }^{4}$ In contrast, in case of abnormal MRI studies, the distribution and morphology of lesions predicts the risk of a second clinical event with high sensitivity and specificity. ${ }^{6}$ Furthermore, the presence of oligoclonal bands in pediatric ON increases the risk of MS. ${ }^{7}$

Pediatric MS is defined by 2 or more non-ADEM episodes of presumed inflammatory demyelinating origin, separated by $>30$ days and involving more than one CNS site or a first nonencephalitogenic episode with an MRI fulfilling the McDonald criteria for dissemination in space (DIS) and with a follow-up MRI showing a new enhancing or nonenhancing lesion consistent with the 2010 dissemination in time (DIT) criteria. In children $\geq 12$ years of age, a single, non-ADEM episode with MRI lesions fulfilling the $2010 \mathrm{McD}$ anald criteria for DIS and DIT may also satisfy the criteria for pediatric MS. In a child with an ADEM attack that is followed by a second nonADEM event, 3 or more months after the first episode, and new MRI lesions that fulfill the 2010 criteria for DIS and DIT, the diagnosis of pediatric MS can be assigned. ${ }^{1}$
$\mathrm{ON}$ or TM are the main presenting features of NMOSD. ${ }^{8}$ Other core clinical characteristics comprise an acute area postrema syndrome (intractable hiccups or vomiting), an acute brainstem syndrome, or symptomatic diencephalic (symptomatic narcolepsy) or cerebral syndrome with NMOSD-typical MRI lesions in conjunction with aquaporin-4 (AQP4) antibodies. ${ }^{9}$ While many clinical, imaging, and CSF features are similar to those found in adult NMOSD, some caveats remain. In particular, longitudinally extensive transverse myelitis (LETM) may be less specific for pediatric NMO, since LETM also may be found in pediatric MS, as well as in monophasic ADEM. ${ }^{9,10}$ Recently, pediatric cases with the clinical picture of ADEM, accompanied by the presence of AQP4 antibodies, also have been described. ${ }^{11}$

Among children with ADS, a subgroup of patients shows serologic evidence for myelin oligodendrocyte glycoprotein (MOG) antibodies. Children with MOGassociated demyelinating CNS disease may present with various clinical features, including ADEM, ADEM followed by ON, or NMOSD., ${ }^{9,12,13}$ MOG-associated ADEM in children shows a uniform radiologic pattern with large, hazy lesions, the absence of abnormal MRI features, and involvement of more CNS areas including spine LETM.

Recent studies showed that the presence of MOG antibodies argues against a further diagnosis of MS. ${ }^{14}$ However, the long-term evolution of children with MOG antibodies needs further prospective evaluation.

\section{RED FLAGS SUGGESTING AN ALTERNATIVE DIAGNOSIS IN CHILDREN WITH ADS In children} with an acute demyelinating event, the following red flags should challenge the diagnosis of ADS: onset before age 1 year; history of developmental delay; consanguineous family; family history of severe, acute neurologic symptoms; gradual progression of symptoms; and multisystemic involvement (table 2). Unusual MRI features that are infrequent in ADS are a single supratentorial white matter lesion, symmetric white matter involvement, or lesions restricted solely to the brainstem and basal ganglia (table 3). 
Table 2 Clinical red flags for conditions other than acquired demyelinating syndromes

\begin{tabular}{|c|c|}
\hline Condition & Clinical red flags \\
\hline Multisystemic involvement & $\begin{array}{l}\text { HLH, Behçet disease, CNS vasculitis, SLE, mitochondrial diseases, sarcoidosis, Sjögren } \\
\text { syndrome, LCH, infections }\end{array}$ \\
\hline Spastic paraplegia & $\begin{array}{l}\text { SLE, Sjögren syndrome, Lyme disease, West Nile virus, vitamin } B_{12} \text { deficiency, spinal cord } \\
\text { tumor/ischemia/AVM/trauma, familial spastic paraplegia, AMN, Krabbe disease, Alexander } \\
\text { disease }\end{array}$ \\
\hline $\begin{array}{l}\text { Protracted headache } \pm \\
\text { stroke-like episodes }\end{array}$ & $\begin{array}{l}\text { SLE, MELAS, MERFF, HIV, malignancy, CADASIL, HLH, CNS vasculitis, Fabry disease, Susac } \\
\text { syndrome }\end{array}$ \\
\hline Epilepsy & Mitochondrial diseases, $\mathrm{B}_{12}$ metabolism disorders \\
\hline Ataxia & NPC, SCA, PDH \\
\hline Extrapyramidal symptoms & $\begin{array}{l}\text { Anti-NMDAR encephalitis, Wilson disease, mitochondrial diseases, biotinidase deficiency, } \\
\text { biotin-responsive basal ganglia disease, LBSL }\end{array}$ \\
\hline Psychiatric symptoms & $\begin{array}{l}\text { Primary CNS angiitis, anti NMDAR encephalitis, } B_{12} \text { metabolism disorders, mitochondrial } \\
\text { disorders, SLE, Susac syndrome }\end{array}$ \\
\hline $\begin{array}{l}\text { Progressive disease with } \\
\text { dementia }\end{array}$ & Leukodystrophies, mitochondrial diseases \\
\hline Hypothalamic dysfunction & Sarcoidosis, LCH \\
\hline Cranial nerve neuropathies & Krabbe disease, MLD, Alexander disease, Lyme disease, sarcoidosis \\
\hline Peripheral neuropathy & Krabbe disease, MLD, mitochondrial disorders, ALD/AMN \\
\hline Sensorineural hearing loss & Susac syndrome, mitochondrial diseases \\
\hline Optic neuropathy & LHON, MELAS, MERFF, OPA-1 \\
\hline
\end{tabular}

Abbreviations: $\mathrm{ALD}=$ adrenoleukodystrophy; $\mathrm{AMN}$ = adrenomyeloneuropathy; $\mathrm{AVM}$ = arteriovenous malformation; $\mathrm{CADASIL}=$ cerebral autosomal dominant arteriopathy with subcortical infarcts and leukoencephalopathy; $\mathrm{LBSL}=$ leukoencephalopathy with brainstem and spinal cord involvement and elevated lactate; $\mathrm{HLH}=$ hemophagocytic lymphohistiocytosis; $\mathrm{LCH}=$ Langerhans cell histiocytosis; LHON = Leber hereditary optic neuropathy; MELAS = mitochondrial encephalomyelopathy with lactic acidosis and stroke; MERFF = myoclonic epilepsy with ragged red fibers; MLD = metachromatic leukodystrophy; NMDAR = NMDA receptor; $\mathrm{NPC}=$ Niemann-Pick C; OPA = optic atrophy; PDH = pyruvate dehydrogenase deficiency; $\mathrm{SCA}=$ spinocerebellar ataxia; $\mathrm{SLE}=$ systemic lupus erythematosus.

INFLAMMATORY DISEASES OF THE WHITE MATTER OTHER THAN ADS CNS vasculitis. CNS vasculitis is one of the most challenging differential diagnoses of ADS and is divided into secondary forms, which are part of a systemic disease or primary angiitis of the CNS (PACNS) (table 4).

Presenting symptoms are often nonspecific, with headache, confusional state, or behavioral alteration reminiscent of psychosis, depression, cognitive decline, seizures, or focal neurologic signs. Although imaging findings in CNS vasculitis can be indistinguishable from various forms of ADS (e.g., TM, $\mathrm{MS}$ ), several findings are characteristic for CNS vasculitis, such as leptomeningeal and vessel wall enhancement, in combination with atrophic areas suggestive of previous events (figure, E). ${ }^{15} \mathrm{MRI}$ and even conventional angiography can be normal despite neuropathologic evidence of CNS vasculitis. ${ }^{15}$ Diagnosis may need to be confirmed by brain biopsy.

Systemic lupus erythematosus (SLE). General symptoms of SLE include a malar rash, arthritis, and fever. Neurologic and psychiatric symptoms often develop during the course of the disease but can also be the first signs of the disease. ${ }^{16}$ The diagnosis is established considering the involvement of different organ systems in combination with anemia, thrombocytopenia, and the presence of antibodies to double-stranded DNA, or high antiphospholipid antibodies. Few studies exist describing the MRI findings in children with SLE, which can include nonspecific T2 signal alterations in particular at the beginning of the disease, larger areas of demyelination, and TM in addition to cortical and subcortical strokes. $^{16,17}$

Behçet disease. Behçet disease is a systemic disorder with neurologic symptoms in $20 \%$ of pediatric cases. ${ }^{18}$ The neurologic presentations can be variable and the sole presenting sign of the disease. The neurologic manifestations are often linked to high intracranial pressure due to cerebral venous sinus thrombosis, inflammation of the white matter, or stroke due to vasculitis. The course of the disease is relapsing, sometimes despite treatment. Cerebral MRI can demonstrate venous sinus thrombosis and less often white matter lesions reminiscent of MS, which is more common in adults. ${ }^{18} \mathrm{CSF}$ studies are normal in up to $50 \%$ of cases.

Primary angiitis of the CNS. The diagnosis of PACNS is based on 3 criteria: (1) an acquired neurologic deficit, (2) angiographic or histologic evidence of cerebral vasculitis, and (3) absence of systemic 
Table 3 Imaging features such as preferred location and additional features of disorders other than acquired demyelinating syndromes

Region of extensive white matter involvement

Frontal white matter

Parieto-occipital

Temporal

Periventricular

Periventricular sparing

Basal ganglia

Thalamus

Brainstem

Involvement of white

matter tracts

Corticospinal

Dorsal column

Subcortical U-fibers

External capsule/insula

Corpus callosum

Spinal cord

Spinal cord hyperintensity

Spinal cord atrophy

Additional features

Meningeal enhancement

Cysts

Calcifications on CT

Gadolinium enhancement

Cortical infarcts

Lacunar infarcts

\section{Conditions to consider}

Alexander disease, MLD, MLC, variant ALD

ALD, Krabbe disease, peroxisomal disorders, mitochondrial diseases (MELAS, POLG1)

CADASIL, AGS, CMV, MLC

HLH, MLD, LBSL (sparing temporal lobes), VWMD (diffuse), CADASIL

KSS

Mitochondrial disorders (e.g., Leigh), biotinidase deficiency, Wilson disease, bilateral striatal necrosis, biotin-responsive basal ganglia disease, AGS

Malignancies, hepatic encephalopathy, ANE, LTBL, Fabry disease (pulvinar)

Rhomboencephalitis, TB, Behçet, Alexander disease, LBSL, LTBL, malignancies

ALD/AMN, Krabbe disease, ALSP

LBSL, vitamin $\mathrm{B}_{12} /$ copper deficiency

Canavan, KSS

ANE, CADASIL

Susac syndrome, Krabbe

Sarcoidosis, SLE, CLIPPERS, infections (WNV, Lyme)

LBSL, Alexander disease, APBD

Sarcoidosis, chronic meningitis, Susac syndrome, CNS vasculitis, CNS lymphoma, Lyme neuroborreliosis

Congenital infections, Alexander (frontal), MLC (frontal, temporal), VWMD, AGS mitochondrial disease

AGS, congenital infection, mitochondrial disease, Cockayne syndrome

Abscess, ALD/AMN, Alexander disease, ANE

CNS vasculitis, MELAS

SLE, CADASIL, Susac syndrome

Abbreviations: AGS = Aicardi-Goutières syndrome; ALD = adrenoleukodystrophy; ALSP = adult-onset leukoencephalopathy with axonal spheroids and pigmented glia; $\mathrm{AMN}=$ adrenomyeloneuropathy; $\mathrm{ANE}=$ acute necrotizing encephalopathy; $\mathrm{APBD}=$ adult polyglucosan body disease; CADASIL = cerebral autosomal dominant arteriopathy with subcortical infarcts and leukoencephalopathy; CLIPPERS = chronic lymphocytic inflammation with pontine perivascular enhancement responsive to steroids; $\mathrm{CMV}$ = cytomegalovirus; $\mathrm{HLH}=$ hemophagocytic lymphohistiocytosis; $\mathrm{KSS}=$ Kearns-Sayre syndrome; LBSL = leukoencephalopathy with brainstem and spinal cord involvement and elevated lactate; LTBL = leukoencephalopathy with thalamus and brainstem involvement and lactate elevation; MELAS = mitochondrial encephalomyelopathy with lactic acidosis and stroke; MLC = megalencephalic leukoencephalopathy with subcortical cysts; MLD = metachromatic leukodystrophy; POLG = polymerase gamma; $\mathrm{SLE}=$ systemic lupus erythematosus; TB = tuberculosis; VWMD = vanishing white matter disease; WNV = West Nile virus.

vasculitis. ${ }^{19}$ PACNS affects the medium-sized and small vessels and remains a rare and difficult diagnosis. Clinical presentations in small vessel disease include seizures, headache, and cognitive decline but also ON or TM. ${ }^{19}$ In children with small vessel disease, systemic signs of inflammation are often present, such as an elevated erythrocyte sedimentation rate, chronic reactive protein, or von Willebrand factor, which has been suggested as a clinical marker of disease activity. ${ }^{20} \mathrm{CSF}$ studies often reveal an elevated opening pressure, pleocytosis, and increased protein levels. ${ }^{21}$ MRI may demonstrate white matter lesions reminiscent of $\mathrm{ADS}$ and normal magnetic resonance angiography. A definitive diagnosis requires brain biopsy encompassing the meninges, gray matter, and white matter, ideally prior to initiation of glucocorticoid treatment. ${ }^{15}$ Medium-sized vessel disease usually presents with a stroke or TIA.

Neurosarcoidosis. Granulomatous disorders such as sarcoidosis are often included in the differential diagnosis of ADS. Although selected cases have been described, children rarely present with sole neurologic symptoms such as aseptic meningitis, 
Table 4 Selected diseases mimicking an acute demyelinating syndrome with clinical clues and recommended workup that should be tailored according to clinical symptoms and likelihood of an alternative diagnosis

\section{Conditions}

Systemic lupus

erythematosus

Behçet disease

Neurosarcoidosis

Isolated or primary
angiitis of the CNS

Hemophagocytic

lymphohistiocytosis

Immunodeficiency

syndromes (e.g., XLP, NK)

Neuroborreliosis

Progressive multifocal

leukoencephalopathy

Acute encephalopathies with

autoantibodies

Steroid-responsive

encephalopathy associated

with autoimmune thyroiditis

\section{Acute cerebellitis}

Guillain-Barré syndrome and Bickerstaff brainstem encephalitis

Solid tumors (astrocytoma, glioma, oligodendroglioma, ependymoma)

CNS lymphoma

Langerhans cell histiocytosis

Neurometabolic diseases (general)

Leber hereditary optic neuropathy

Kearns-Sayre syndrome, Leigh syndrome, POLG-related disorders

Leukoencephalopathy with brainstem and spinal cord involvement and lactate elevation

Acute necrotizing encephalopathy

Biotin responsive basal ganglia disease

Migraine

\section{Clinical clues}

Rash, arthralgias, headache, stroke, neuropsychiatric symptoms, cognitive changes, movement disorder, spastic paraplegia

Optic neuritis, uveitis, rash, arthralgias, oral/genital ulcers, spastic paraplegia, stroke, cerebrovenous sinus thrombosis

Basilar meningitis, uveoparotid fever (uveitis, parotid swelling, facial nerve swelling), cranial neuropathy, raised ICP, seizure, cognitive changes, peripheral neuropathy, spastic paraplegia

Headache, stroke, seizures, encephalopathy, visual abnormalities, cognitive changes

Fever, seizures, meningismus, motor deficit, affected sibling/consanguinity

Early: Meningoradiculitis, cranial neuritis (e.g., facial palsy), meningitis, plexus neuritis, mononeuritis multiplex, erythema migrans

Late: Encephalomyelitis, myelitis, encephalitis, vasculitis, chronic meningitis

Immunosuppressed individual, hemiparesis, dysphasia, ataxia, cortical visual deficits, cognitive changes, seizures, headaches

Seizures, neuropsychiatric symptoms, orofacial dyskinesias and sleep disturbances, autonomic dysfunction

Encephalopathy, seizures, focal neurologic signs, neuropsychiatric features

Ataxia, headache, brainstem syndromes

Ascending sensorimotor neuropathy, ataxia, areflexia, extraocular movement abnormalities

Typically monofocal neurologic deficits, persisting symptoms, pain

Headache, ataxia, seizures, hemiparesis

Abnormalities of the hypothalamic-pituitary axis behavior changes, seizures, visual deficits, headaches

Variable degree of acute or episodic neurologic deficits, neurologic regression

Unilateral or bilateral severe vision loss, abnormal retinal

Kearns-Sayre syndrome: Extraocular movement abnormalities pigmentary retinopathy, cardiac conduction abnormalities, myopathy, ataxia

Leigh syndrome: Psychomotor regression, failure to thrive, hypotonia, ataxia

POLG: Acute encephalopathy, seizures, cognitive decline, stroke-like episodes

Progressive spasticity, ataxia and dorsal column dysfunction

Acute encephalopathy, seizures

Acute encephalopathy with dystonia, seizures

Recurrent headache meeting international headache classification
Consider the following investigations (in addition to MRI brain/spine)

ESR, complement (C3, C4), ANA, dsDNA, rheumatology evaluation

Examination: Oral and genital ulcers; skin pathergy test

Serum/CSF ACE, calcium, ESR, IgG levels, CSF studies (flow cytometry CD4:CD8), CXR \pm high-resolution $\mathrm{CT}$, bronchoalveolar lavage

ESR, CRP, von Willebrand factor, MRA, VZV antibodies, CSF studies, conventional angiogram, brain biopsy

$\mathrm{CBC}$, triglycerides, ferritin, bone marrow aspiration, CSF studies, genetic testing

Serum antibodies against Borrelia burgdorferi, CSF studies with cell count (lymphocytic), Al IgG and PCR B burgdorferi

CSF PCR for JC virus

Serum/CSF NMDA, GABA-A, glycine receptor antibodies, EEG

Antithyroid peroxidase, antithyroglobulin antibodies

CSF studies

CSF studies, GQ1 antibodies, NCS

CSF analysis including cytology, lesional biopsy, tumor specific testing

CSF analysis including cytology, lesional biopsy, CSF immunotyping

CSF studies, lesional biopsy, CBC, liver enzymes, immunoglobulin levels, ESR, bone marrow aspiration/biopsy, BRAF gene mutation

Muscle/skin biopsy, MRS, CSF/serum lactate, aminoacids, NCS, ophthalmology evaluation

Mutation in mtDNA m.3460G $>$ A,

$\mathrm{m} \cdot 11778 \mathrm{G}>\mathrm{A}$, or $\mathrm{m} \cdot 14484 \mathrm{~T}>\mathrm{C}$ in $90 \%$

Mitochondrial genetic testing, POLG mutation

DARS2 mutation

Liver enzymes, CSF studies, RANBP2 mutation

SLC19A3 mutation

Clinical history, exclusion of other diseases

Abbreviations: $\mathrm{ACE}=$ angiotensin-converting enzyme; $\mathrm{Al}=$ antibody index; $\mathrm{ANA}=$ anti-nuclear antibody; $\mathrm{CBC}=$ complete blood count; $\mathrm{CRP}=\mathrm{C}$-reactive protein; $\mathrm{CXR}=$ chest X-ray; dsDNA = double-stranded DNA; ESR = erythrocyte sedimentation rate; $\mathrm{GABA}=\gamma$-aminobutyric acid; ICP $=$ intracranial pressure; IgG = immunoglobulin G; MRA = magnetic resonance angiography; MRS = magnetic resonance spectroscopy; $\mathrm{NCS}=$ nerve conduction study; NK = natural killer; POLG $=$ polymerase $\gamma ; \mathrm{VZV}=$ varicella-zoster virus; $\mathrm{XLP}=\mathrm{X}$-linked lymphoproliferative syndrome. 
Figure Cerebral and spinal MRI of children with episodes of an acquired demyelinating syndrome and examples of diseases than can initially present with similar symptoms
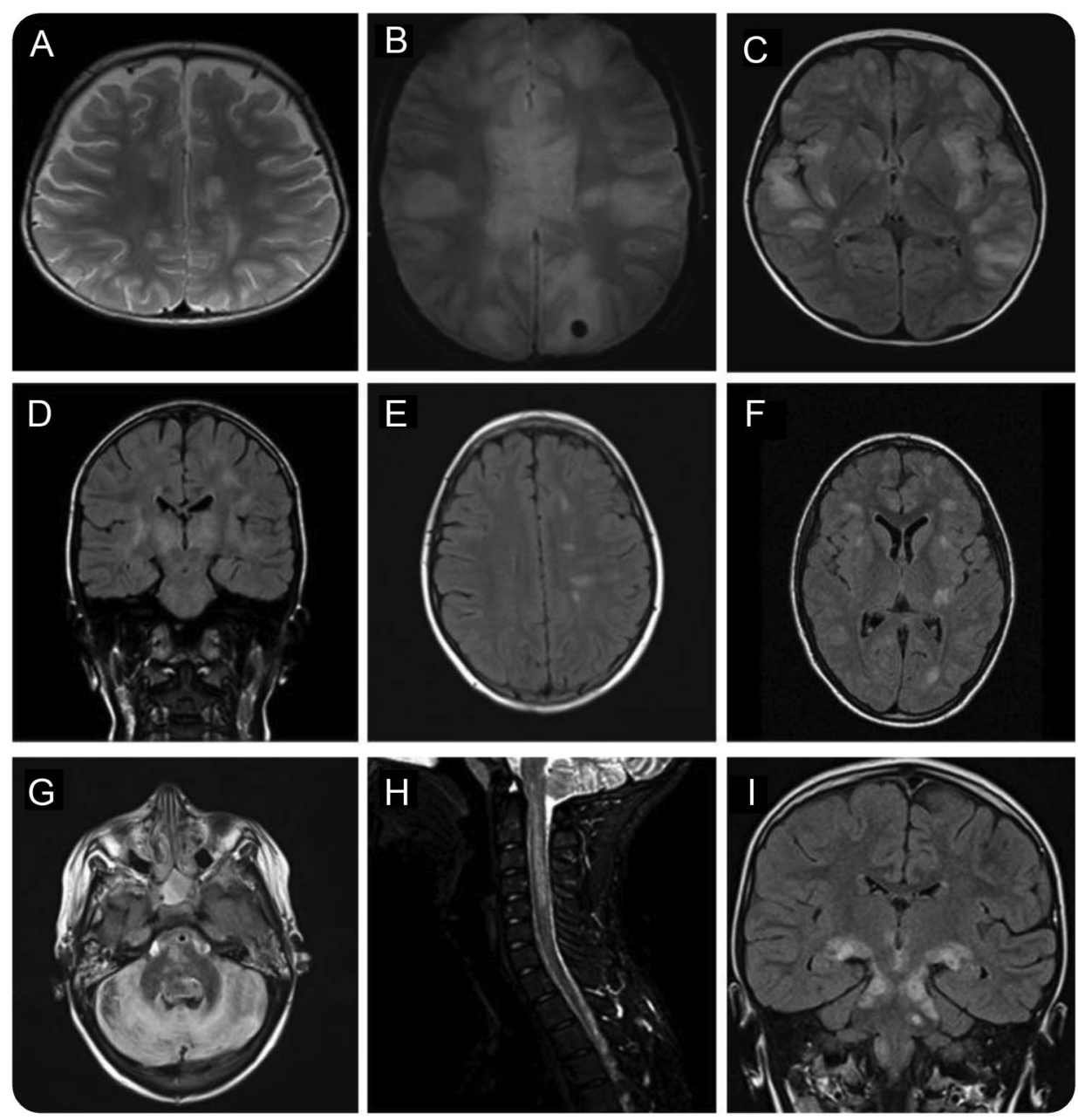

(A) MRI of a 7-year-old girl with a fulminant episode of acute disseminating encephalomyelitis (ADEM) characterized by encephalopathy, respiratory distress, focal neurologic signs, and myelin oligodendrocyte glycoprotein antibodies shows hazy bilateral and large signal intensities in the subcortical white matter. (B) Cerebral MRI of a 15-year-old girl who developed an acute hemorrhagic leukoencephalopathy after a urinary infection with Escherichia coli. (C) MRI of a 4-year-old boy with sudden onset of ataxia and drowsiness shows multiple subcortical white matter lesions reminiscent of ADEM. The patient was diagnosed with an X-linked immunoproliferative disease due to a mutation in the SH2 domain protein 1A. (D) Cerebral MRI of a 10-year-old boy who presented with irritability, vomiting, double vision, and vertigo shows hazy bilateral lesions in the white matter and thalami. A high titer of aquaporin-4 antibodies was detected and he was diagnosed with neuromyelitis optica spectrum disorder. (E) A 12-year-old girl with episodes of impaired unilateral vision and hemiparesis initially diagnosed with multiple sclerosis had multiple small white matter lesions on the left side of her brain. Magnetic resonance angiography and CSF pleocytosis pointed to the diagnosis of CNS vasculitis. After further stroke-like episodes, she was started on cyclophosphamide, leading to significant improvement. (F) A 13-year-old boy presented with bilateral motor weakness, ataxia, and unilateral peripheral facial nerve palsy. His initial MRI with large bilateral lesions also affecting the corpus callosum pointed to a multifocal clinically isolated syndrome. Subsequent neurophysiologic studies and response to IV immunoglobulin confirmed the diagnosis of Guillain-Barré syndrome with white matter lesions. (G) A 13-year-old girl presented with nausea, vomiting, and loss of consciousness due to a prominent cerebellitis affecting white matter and cortical structures of the cerebellum. (H) A 16-year-old boy was admitted with weakness in both arms, which had gradually evolved during the last 3 weeks. MRI of the spine reveals an edematous swelling of the myelin extending more than 3 segments. CSF studies and serologic studies showed immunoglobulin $\mathrm{M}$ and an intrathecal synthesis of immunoglobulin $\mathrm{G}$ antibodies against Borrelia burgdorferi. (I) A 4-year-old girl developed somnolence, motor weakness, and gait ataxia after a viral infection. Her MRI shows symmetric lesions in the midbrain extending into the basal ganglia suggestive of acute necrotizing encephalitis. She had a mild CSF pleocytosis and a good response to IV steroids. Further workup revealed a mutation in the RANBP2 gene.

cranial neuropathy, or space-occupying lesions. Most children have systemic symptoms at initial presentation such as arthritis or swelling of the parotid gland. ${ }^{22}$ In CSF, elevated opening pressure, lymphocytosis, and elevated protein are common.
Brain MRI can demonstrate granulomata in the parenchyma, frequently enhancing after gadolinium administration and periventricular white matter lesions. A lesional biopsy may be required to establish the diagnosis. 
Genetic defects affecting the immune response. Hemophagocytic lymphohistiocytosis (HLH) represents a group of inherited immunodeficiencies, including familial HLH, Griscelli syndrome type 2, and X-linked lymphoproliferative syndrome, which are caused by defects in natural killer cells and CD8 T-cell cytotoxicity. Secondary forms are found in combination with infections, cancer, and other autoimmune disorders. ${ }^{23}$ Primary HLH remains asymptomatic until a viral infection, such as Epstein-Barr virus, triggers the activation of $\mathrm{CD} 8+\mathrm{T}$ lymphocytes and macrophages. Initial symptoms can be restricted to the CNS and consist of seizures, impaired consciousness, meningism, and focal motor deficits. ${ }^{24}$ Biological markers indicating $\mathrm{HLH}$ are pancytopenia, low fibrinogen levels, and elevated liver enzyme and ferritin levels. Signs of hemophagocytosis are detectable on CSF/blood/bone marrow smears in two-thirds of children. CSF results can be abnormal in half of all children demonstrating high protein and increased cell count. ${ }^{25}$ Cerebral MRI can demonstrate symmetric white matter lesions sometimes difficult to differentiate from ADEM in addition to signal hypointensity on $\mathrm{T} 1$ sequences (figure, $\mathrm{C}$, table 4 ).

Infections. Neuroborreliosis. In general, children with Lyme neuroborreliosis present with unilateral or bilateral 7th nerve palsy or meningitis. Most cases occur in the late spring and summer and in the 5- to 14-year age group with neurologic involvement in $5 \%-15 \%$ of children. ${ }^{26}$ Diagnosis relies on the presence of a CSF pleocytosis and an intrathecal synthesis of immunoglobulin $\mathrm{G}(\mathrm{IgG})$ and immunoglobulin $\mathrm{M}$ antibodies against the pathogen. Infections with Borrelia burgdorferi affecting the neuroaxis can present as $\mathrm{ON}$ or TM with or without radiologic evidence of LETM (figure, $\mathrm{H})$ or CNS vasculitis with white matter lesions and focal neurologic signs. ${ }^{27}$

Progressive multifocal leukoencephalopathy (PML). JC virus is a ubiquitous polyomavirus that causes PML, a frequently fatal demyelinating disease, in particular in immunocompromised patients. Presentation can be insidious with cognitive decline or more rapid with seizures and gait abnormalities. MRI changes show T2-hyperintense lesions in the frontoparietal white matter. Contrast enhancement is unusual. Diagnosis is confirmed on finding JC virus DNA in the CSF. ${ }^{28}$

Autoantibody-mediated diseases associated with white matter changes. Several antibodies against neuronal surface antigens have been detected in recent years. Although they commonly present with encephalitis, psychiatric symptoms, refractory seizures, or movement disorders, some of them have been shown to be associated with white matter lesions on MRI. Glycine receptor antibodies usually observed in stiffperson syndrome have been found in children and adults with ON. ${ }^{29}$ CSF antibodies to the inhibitory $\gamma$-aminobutyric acid A receptor have been discovered in children with encephalitis and white matter changes. ${ }^{30}$ Titulaer et al. $^{31}$ recently reported adults with NMDA receptor encephalitis who subsequently developed demyelinating syndromes associated with MOG or AQP4 antibodies.

Steroid-responsive encephalopathy is characterized by the combination of anti-thyroperoxidase antibodies with symptoms such as acute encephalitis with seizures, focal neurologic signs, and neuropsychiatric symptoms. Brain MRI lesions can involve the white and gray matter in particular of the subcortical regions and brainstem, often in a symmetrical fashion, which are distinct from MS or ADEM in most cases. ${ }^{32}$

Acute inflammatory episodes with demyelination other than ADS. Acute cerebellitis is defined by symptoms and MRI changes related primarily to the cerebellum and signs of inflammation in the CSF such as an elevated CSF cell count. Acute cerebellitis can lead to severe brainstem dysfunction. In less acute forms, unilateral or bilateral signal changes of the cerebellar cortex around the dentate nucleus or white matter are observed (figure, G). In children in whom the MRI additionally demonstrates supratentorial, brainstem, or spinal lesions, an inflammatory process such as ADEM should be considered.

Miller Fisher syndrome, Guillain-Barré syndrome (GBS), and Bickerstaff brainstem encephalitis (BBE) have been considered part of a clinical spectrum with a range of symptoms involving the CNS and peripheral nervous system. BBE reflecting the severe end of the spectrum is characterized by encephalopathy and signs of brainstem dysfunction in addition to hyporeflexia or areflexia. ${ }^{33}$ CSF shows a normal cell count but an elevated protein. Increased titers of antiGQ1b-IgG antibodies can be used in support of diagnosis. White matter lesions affecting the brainstem or other regions have been reported in up to $1 / 3$ of patients with GBS and BBE (figure, F). Timely diagnosis is important because of the severity and usually prompt response to immunotherapy. ${ }^{33}$

TUMORS Solid tumors of the CNS. Tumors such as oligodendroglioma, ependymoma, low-grade astrocytoma, and high-grade glioma may initially lead to the incorrect diagnosis of ADS. Important signs are a gradual development of clinical symptoms, the absence of multiple lesions on initial MRI, and lesions that also involve cortical areas extending to other anatomical areas. Disseminated astrocytoma can have spatial dissemination. CSF cell count can be elevated in addition to a high protein. It is recommended to always include a cytospin CSF analysis in the workup of a child with an unusual clinical presentation and inconclusive MRI findings. 
Primary CNS lymphoma (PCNSL). PCNSL is often included in the differential diagnosis of an acute demyelinating event. PCNSL is an exceedingly rare but challenging diagnosis in children. Localization of PCNSL is often in the cerebral hemispheres but can be at any anatomical site including the leptomeninges. Therefore, symptoms at presentation are highly variable, including headache, visual problems, ataxia, seizures, and hemiparesis. MRI often reveals lesions in the cerebral hemispheres, basal ganglia, cerebellum, or brainstem, occasionally with meningeal enhancement. ${ }^{34}$ Diagnosis is confirmed by brain biopsy or immunophenotypic analyses of CSF. The majority of children reported in the literature have a CNS nonHodgkin lymphoma. ${ }^{34}$

Langerhans cell histiocytosis ( $\mathbf{L C H})$. LCH is a multisystem disease and neurologic symptoms are primarily related to solitary or multifocal lesions in meninges and other areas of the CNS including the brainstem and cerebellum. ${ }^{35}$ In the absence of a clinical history of $\mathrm{LCH}$, an isolated CNS lesion presents a diagnostic challenge. MRI findings in these patients include symmetric hyperintense signals in T1-weighted images in the cerebellar gray and white matter, cerebellar atrophy, T2-weighted hyperintense changes in the pons, and T1-weighted hyperintensity of the globus pallidum.

NEUROMETABOLIC DISEASES A range of neurometabolic conditions are associated with white matter changes in the CNS, many of which present acutely in the context of a nonspecific illness with encephalopathy and neurologic symptoms, reminiscent of ADEM. MRI changes that are symmetrical and involve the striatum should alert the clinician to investigate in particular treatable diseases such as biotin-responsive basal ganglia disease (table 4).

Mitochondrial respiratory chain defects can present with symptoms and MRI findings indistinguishable from ADS. An evaluation of lactate content and of lactate/pyruvate ratio in blood and CSF, spectroscopy focusing on white matter lesions, and a direct study of respiratory chain activity in fibroblasts or muscle biopsy may be required in addition to genetic testing to establish the diagnosis. Leber hereditary optic neuropathy, caused by point mutations in mtDNA, deserves special consideration because of its associations with MS and ON. ${ }^{36,37}$ Other important mitochondrial disorders include Leigh syndrome, Kearns-Sayre syndrome, or leukoencephalopathy with brainstem and spinal cord involvement. ${ }^{38}$ The latter disease can present in a previously healthy child with pyramidal and cerebellar dysfunction. MRI abnormalities include spotty, periventricular white matter changes, as well as cerebellar white matter, brainstem, and spinal cord imaging similar to LETM. Further episodes can occur and respond to steroids. ${ }^{38}$

Two other diseases affecting cellular energy metabolism are biotin-responsive basal ganglia disease (BBGD), which is due to a SLC19A3 mutation, and acute necrotizing encephalopathy (ANE). In the latter, a heterozygous mutation in RANBP2 is found in familial and recurrent ANE, a rapidly progressive encephalopathy that typically occurs in previously healthy children between 6 and 18 months of age. ${ }^{39}$ Typically, there is a prodrome of a febrile illness prior to the acute phase of encephalopathy with loss of consciousness and seizures. Mortality and neurologic sequelae are common in particular in cases not treated promptly with steroids (figure, I). Liver transaminases are mildly elevated with increased CSF protein in the absence of pleocytosis. MRI demonstrates symmetrical involvement of the thalamus, with additional regions affected including the periventricular white matter, putamen, internal capsule, brainstem, and cerebellum. ${ }^{39}$

BBGD should be considered in the context of an ADEM-like presentation and symmetrical basal ganglia involvement and treated immediately with biotin and thiamin.

Leukodystrophies such as Alexander disease, X-linked adrenoleukodystrophy, or metachromatic leukodystrophy can present at various ages with atypical presentations and MRIs, but due to their progressive nature and MRI patterns, should be differentiated easily from acute demyelinating syndromes.

MIGRAINE The detection of nonspecific incidental white matter changes in children with headache, or more specifically migraine, is now well-established. Different series demonstrate a similar rate of up to $6 \%$ of white matter hyperintensities with a trend for a higher prevalence in migraine with aura. ${ }^{40}$ Lesions are only supratentorial. The distinction from CIS is usually on clinical grounds and on differences in signal intensity, localization, and absence of gadolinium enhancement.

DISCUSSION Precise and detailed description of the clinical picture, along with MRI and CSF studies, allow the characterization of the different ADS in the majority of children. Nevertheless, a proportion of cases are due to a broad spectrum of other inflammatory disorders of the white matter, primary CNS tumors, and neurometabolic diseases. The clinician therefore has to be aware of the different forms of ADS, the risk factors for a chronic-relapsing course, and features that indicate an alternative diagnosis.

\section{AUTHOR CONTRIBUTIONS}

Kevin Rostasy: concept, outline, and writing of the manuscript. Barbara Bajer-Kornek: writing of the manuscript. Sunita Venkateswaran: writing of the manuscript and tables. Cheryl Hemingway: writing of the 
manuscript. Marc Tardieu: concept, outline, and writing of the manuscript. All authors contributed extensively to the editing and consensusfinding process of all sections of the manuscript.

\section{STUDY FUNDING}

This supplement is made possible by funding from the MS Cure Fund, Danish MS Society, German MS Society, Italian MS Association, MS International Federation, MS Research Foundation (Netherlands), National MS Society (USA) and Swiss MS Society.

\section{DISCLOSURE}

The authors report no disclosures relevant to the manuscript. Go to Neurology.org for full disclosures.

Received August 19, 2015. Accepted in final form February 4, 2016.

\section{REFERENCES}

1. Krupp LB, Tardieu M, Amato MP, et al; International Pediatric Multiple Sclerosis Study Group. International Pediatric Multiple Sclerosis Study Group criteria for pediatric multiple sclerosis and immune-mediated central nervous system demyelinating disorders: revisions to the 2007 definitions. Mult Scler 2013;19:1261-1267.

2. Ketelslegers IA, Neuteboom RF, Boon M, CatsmanBerrevoets CE, Hintzen RQ; Dutch Pediatric MS Study Group. A comparison of MRI criteria for diagnosing ADEM and MS. Neurology 2010;74:1412-1415.

3. Banwell B, Kennedy J, Sadovnick D, et al. Incidence of acquired demyelination of the CNS in children. Neurology 2009;72:232-239.

4. Verhey LH, Branson HM, Shroff MM, et al; Canadian Pediatric Demyelinating Disease Network. MRI parameters for prediction of multiple sclerosis diagnosis in children with acute CNS demyelination: a prospective national cohort study. Lancet Neurol 2011;10:10651073.

5. Ketelslegers IA, Catsman-Berrevoets CE, Neuteboom RF, et al. Incidence of acquired demyelinating syndromes of the CNS in Dutch children: a nationwide survey. J Neurol 2012;259:1929-1935.

6. Kornek B, Schmitl B, Vass K, et al. Evaluation of the 2010 McDonald multiple sclerosis criteria in children with a clinically isolated syndrome. Mult Scler 2012;18:17681774 .

7. Heussinger N, Kontopantelis E, Gburek-Augustat J, et al. Oligoclonal bands predict multiple sclerosis in children with optic neuritis. Ann Neurol 2015;77: 1076-1082.

8. Wingerchuk DM, Banwell B, Bennett JL, et al; International Panel for NMO Diagnosis. International consensus diagnostic criteria for neuromyelitis optica spectrum disorders. Neurology 2015;85:177-189.

9. Baumann M, Sahin K, Lechner C, et al. Clinical and neuroradiological differences of paediatric acute disseminating encephalomyelitis with and without antibodies to the myelin oligodendrocyte glycoprotein. J Neurol Neurosurg Psychiatry 2015;86:265-272.

10. Verhey LH, Branson HM, Makhija M, Shroff M, Banwell B. Magnetic resonance imaging features of the spinal cord in pediatric multiple sclerosis: a preliminary study. Neuroradiology 2010;52:1153-1162.

11. Absoud M, Lim MJ, Appleton R, et al. Paediatric neuromyelitis optica: clinical, MRI of the brain and prognosis. J Neurol Neurosurg Psychiatry 2015;86: 470-472.
12. Huppke P, Rostasy K, Karenfort M, et al. Acute disseminated encephalomyelitis followed by recurrent or monophasic optic neuritis in pediatric patients. Mult Scler 2013; 19:941-946.

13. Rostasy K, Mader S, Hennes E, et al. Persisting myelin oligodendrocyte glycoprotein antibodies in aquaporin-4 antibody negative pediatric neuromyelitis optica. Arch Neurol 2012;69:752-756.

14. Ketelslegers IA, Van Pelt DE, Bryde S, et al. Anti-MOG antibodies plead against MS diagnosis in an acquired demyelinating syndromes cohort. Mult Scler 2015;21:1513-1520.

15. Twilt M, Benseler SM. CNS vasculitis in children. Mult Scler Relat Disord 2013;2:162-171.

16. Muscal E, Robin L, Brey RL. Neurological manifestations of systemic lupus erythematosus in children and adults. Neurol Clin 2010;28:61-73.

17. Benseler SM, Silverman ED. Neuropsychiatric involvement in pediatric systemic lupus erythematosus. Lupus 2007;16:564-571.

18. Uluduz D, Kurtuncu M, Yapici Z, et al. Clinical characteristics of pediatric onset neuro-Behçet disease. Neurology 2011;77:1900-1905.

19. Elbers J, Halliday W, Hawkins C, et al. Brain biopsy in children with primary small-vessel central nervous system vasculitis. Ann Neurol 2010;68:602-610.

20. Cellucci T, Tyrrell PN, Pullenayegum E, Benseler SM. Von Willebrand factor antigen: a possible biomarker of disease activity in childhood central nervous system vasculitis? Rheumatology 2012;51:1838-1845.

21. Hutchinson C, Elbers J, Halliday W, et al. Treatment of small vessel primary CNS vasculitis in children: an openlabel cohort study. Lancet Neurol 2010;9:1078-1084.

22. Anand G, Sin FE, Soilleux E, et al. Isolated paediatric neurosarcoidosis presenting as epilepsia partialis continua: a case report and review of the literature. Eur J Paediatr Neurol 2013;17:429-436.

23. Horne A, Trottestam H, Arico M, et al; Histiocyte Society. Frequency and spectrum of central nervous system involvement in 193 children with hemophagocytic lymphohistiocytosis. Br J Haematol 2008;140:327-335.

24. Deiva K, Mahlaoui N, Beaudonnet F, et al. CNS involvement at the onset of primary hemophagocytic lymphohistiocytosis. Neurology 2012;78:1150-1156.

25. Mahlaoui N, Ouachée-Chardin M, de Saint Basile G, et al. Immunotherapy of familial hemophagocytic lymphohistiocytosis with antithymocyte globulins: a single-center retrospective report of 38 patients. Pediatrics 2007;120: e622-e628.

26. O'Connell S. Lyme borreliosis: current issues in diagnosis and management. Curr Opin Infect Dis 2010;23: 231-235.

27. Baumann M, Birnbacher R, Koch J, Strobl R, Rostásy K. Uncommon manifestations of neuroborreliosis in children. Eur J Paediatr Neurol 2010;14:274-277.

28. Ferenczy MW, Marshall LJ, Nelson CD, et al. Molecular biology, epidemiology, and pathogenesis of progressive multifocal leukoencephalopathy, the JC virus-induced demyelinating disease of the human brain. Clin Microbiol Rev 2012;25:471-506.

29. Martinez-Hernandez E, Sepulveda M, Rostásy K, et al. Antibodies to aquaporin 4, myelin-oligodendrocyte glycoprotein, and the glycine receptor $\alpha 1$ subunit in patients with isolated optic neuritis. JAMA Neurol 2015; $72: 187-193$ 
30. Petit-Pedrol M, Armangue T, Peng X, et al. Encephalitis with refractory seizures, status-epilepticus, and antibodies to the GABAA receptor: a case series, characterization of the antigen and analysis of the effects of antibodies. Lancet Neurol 2014;13:276-286.

31. Titulaer MJ, Hoftberger R, Iizuka T, et al. Overlapping demyelinating syndromes and anti-N-methyl-D-aspartate receptor encephalitis. Ann Neurol 2014;75:411-428.

32. Mamoudjy N, Korff C, Maurey H, et al. Hashimoto's encephalopathy: identification and long-term outcome in children. Eur J Paediatr Neurol 2013;17:280-287.

33. Pavone P, Le Pira A, Greco F, et al. Bickerstaff s brainstem encephalitis $(\mathrm{BBE})$ in childhood: rapid resolution after intravenous immunoglobulins treatment. Eur Rev Med Pharmacol Sci 2014;18:2496-2499.

34. Abla O, Weitzman S, Blay JY, et al. Primary CNS lymphoma in children and adolescents: a descriptive analysis from the International Primary CNS Lymphoma Collaborative Group (IPCG). Clin Cancer Res 2011;17: $346-352$.
35. Savardekar A, Tripathi M, Bansal D, Vaiphei K, Gupta SK. Isolated tumorous Langerhans cell histiocytosis of the brainstem: a diagnostic and therapeutic challenge. J Neurosurg Pediatr 2013;12:258-261.

36. Palace J. Multiple sclerosis associated with Leber's hereditary optic neuropathy. J Neurol Sci 2009;286:24-27.

37. Weisfeld-Adams JD, Katz Sand IB, Honce JM, et al. Differential diagnosis of Mendelian and mitochondrial disorders in patients with suspected multiple sclerosis. Brain 2015;138:517-539.

38. Wolf N, Toro C, Kister I, et al. DARS-associated leukoencephalopathy can mimic a steroid-responsive neuroinflammatory disorder. Neurology 2015;84:226-230.

39. Neilson DE, Adams MD, Orr CM, et al. Infection-triggered familial or recurrent cases of acute necrotizing encephalopathy caused by mutations in a component of the nuclear pore, RANBP2. Am J Hum Genet 2009;84:44-51.

40. Candee MS, McCandless RT, Moore KR, et al. White matter lesions in children and adolescents with migraine. Pediatr Neurol 2013;49:393-396. 


\section{Neurology}

\section{Differential diagnosis and evaluation in pediatric inflammatory demyelinating disorders}

Kevin Rostasy, Barbara Bajer-Kornek, Sunita Venkateswaran, et al. Neurology 2016;87;:S28-S37

DOI 10.1212/WNL.0000000000002878

\section{This information is current as of August 29, 2016}

\section{Updated Information \& Services}

References

Citations

Subspecialty Collections

Permissions \& Licensing

Reprints including high resolution figures, can be found at: http://n.neurology.org/content/87/9_Supplement_2/S28.full

This article cites 40 articles, 4 of which you can access for free at: http://n.neurology.org/content/87/9_Supplement_2/S28.full\#ref-list-1

This article has been cited by 2 HighWire-hosted articles: http://n.neurology.org/content/87/9_Supplement_2/S28.full\#\#otherartic les

This article, along with others on similar topics, appears in the following collection(s):

Acute disseminated encephalomyelitis

http://n.neurology.org/cgi/collection/acute_disseminated_encephalomy elitis

Multiple sclerosis

http://n.neurology.org/cgi/collection/multiple_sclerosis

Information about reproducing this article in parts (figures,tables) or in its entirety can be found online at:

http://www.neurology.org/about/about_the_journal\#permissions

Information about ordering reprints can be found online:

http://n.neurology.org/subscribers/advertise

Neurology ${ }^{\circledR}$ is the official journal of the American Academy of Neurology. Published continuously since 1951, it is now a weekly with 48 issues per year. Copyright (O 2016 American Academy of Neurology. All rights reserved. Print ISSN: 0028-3878. Online ISSN: 1526-632X.

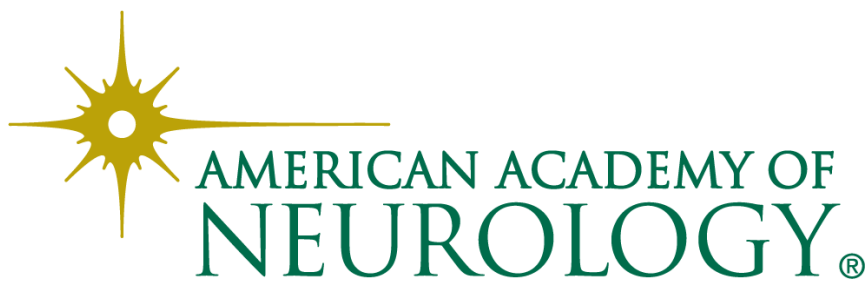

